\title{
Using Rat Navigation Models to Learn Orientation from Visual Input on a Mobile Robot
}

\author{
Brett Browning \\ Carnegie Mellon University, 5000 Forbes Avenue, Pittsburgh, PA, 15213
}

\begin{abstract}
Rodents possess extraordinary navigation abilities that are far in excess of what current state-of-the-art robot agents are capable of. This paper describes research that is part of a larger project aimed at developing a robot navigation system that is capable of robust autonomous navigation in real-time by using biologically plausible constructs inspired from the many neurological and behavioral studies conducted on freely navigating rats. Specifically, this paper discusses the implementation of a ratinspired system that allows a robot to learn and recognize its allocentric orientation based on what it perceives. The system described here is a pragmatic, minimalist implementation of recently proposed models of the rat head direction system. The rat head direction system are collectively, the neural assemblies that neuroscientific studies suggest are responsible for encoding the orientation of the rat's head in a global reference frame. This paper describes an implementation of the system on an autonomous robot that operates in real time, in office environments, and with limited computational hardware. Experiments conducted in a messy laboratory environment with a range of visual features demonstrate that the system, while simple in structure, is able to learn and recognize allocentric orientations after 5 minutes of exploration. The results demonstrate the worth of bridging robotics with biological research and pave the way for developing a more complete, and competent, robot architecture.
\end{abstract}

\section{Keywords}

Adaptation and Learning, Autonomous Robots.

\section{INTRODUCTION}

Navigating rats have been the subjects of neurological and behavioral studies for close to a century. Many, if not all, of the various navigation experiments performed on rats resolutely demonstrate that their navigation abilities far exceed what current state-of-the-art robots are capable of. Moreover, neurological studies over the past thirty years, when combined with behavioral studies are beginning to paint a picture of how rats perform navigation at a neural level. Although many of the details are still incomplete, or worse contradictory, these results have lead numerous researchers to propose models of the rat navigation system and in some cases to simulate these models. The robust,

Permission to make digital or hard copies of all or part of this work for personal or classroom use is granted without fee provided that copies are not made or distributed for profit or commercial advantage and that copies bear this notice and the full citation on the first page. To copy otherwise, or republish, to post on servers or to redistribute to lists, requires prior specific permission and/or a fee. AGENTS'01, May 28-June 1, 2001, Montréal, Québec, Canada. Copyright 2001 ACM 1-58113-326-X/01/0005 ...\$5.00. proficient nature of the rat navigation system suggests that robotics could gain from robotic implementation of rat navigation models. Similarly, neuroscience gains from robotic implementations of proposed models of the rat system. Although some attempts to 'roboticize' rat navigation models have been made (e.g. [2]), much work still remains.

This paper focuses on a robotic implementation of recent models of the so-called rat head direction system. Recent neurological results show that rats have separate neural assemblies for representing their orientation in a world-centered frame. These results have lead researchers to propose a model of the rat head direction (HD) system, which has been progressively refined in the light of more recent data (e.g. [3]). This paper describes the implementation of a HD model inspired from [4] on a real robot using monocular vision as the primary sensor. See [1] for more details on the robot platform.

\section{RATS AND SPACE}

Studies show that rats posses a number of neural assemblies that encode spatial information in different ways. That is, cells that represent encode some spatial property through their firing rate. The most well known, and most studied, examples of such spatial tuning are hippocampal place cells (see [3]) but many other have been found as well. This paper focuses on the rat HD system, an assembly of neurons where the firing rate of each cell appears to encode whether or not the animal is facing a specific direction, called the cells preferred direction. It is believed that the combined activity of many HD cells with different preferred directions prescribes an activity packet reminiscent of a probabilistic distribution encoding the animal's head direction.

Many experiments show that HD cell activity is driven by ideothetic input and bindings with prominent visual cues, although the neural mechanisms behind HD activity remain a mystery. Nonetheless, numerous models have been proposed and some simulated (e.g. [3]). All these models focus on neural structures that lead to the formation of HD cell-like activity packets. To the author's knowledge, no model actually simulates in any realistic way bindings to visual cues. [4] proposed a general hebbian-like mechanism for binding stable views to active HD cells such that a strong connection would form between views observable in one global direction and the corresponding HD cells. To date no one has simulated this approach. This paper works from the general principles proposed by [4] to build a minimalist working robot system.

\section{A MINIMALIST ROBOTIC APPROACH}

This paper focuses on building a robot system that is capable of learning the relationships between what it sees and what direction it is facing. Given the paucity of hard knowledge on the neural mechanisms underlying the HD system and the rat's visual system, one must make engineering judgments to produce a working system. Here a minimalistic approach, building on the proposal of 
[4], has been taken. Thus the system developed attempts to naively bind prominent views to ideothetically driven HD-like activity packets as shown in Figure 1.

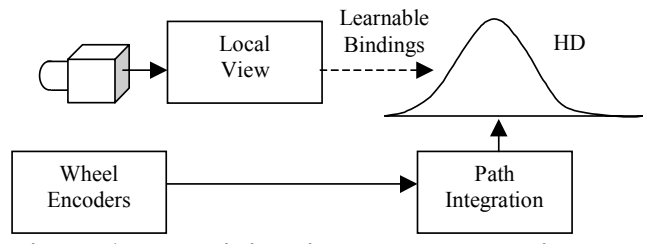

Figure 1. The minimalist robot $\mathrm{HD}$ architecture.

The activity packet in the HD network is generated apriori and shifted using dead-reckoning information derived from the robot encoders. This deviates from many HD model simulations, where plausible activity packet generation is investigated. Given the lack of knowledge on how rodents see, one must become inventive. Most mammals use visual attention mechanisms to decide what they observe. The view system used here is a simplified visual attention system where the robot selects an 'interesting' horizontal viewing angle within the its wider field of view. The $1 \mathrm{D}$ visual attention system used was inspired from [2]. For each frame $(\sim 9 \mathrm{~Hz})$, the average intensity of each column is calculated and the maximum value found. The robot chooses the closest candidate column, those with an average intensity within $70 \%$ of the maximum value, to the prior viewing angle while accounting for the robot's rotation. Periodically shifting the fixation angle helps prevent the robot fixating on one thing for too long. A fovea region is extracted from the viewing angle, which with some filtering becomes the $32 \times 16$ input to the template matching View network. The View network dynamically allocates new nodes for new views if they are observed contiguously for a long enough time and are not currently in the network. Actively matching views are bound to the output of the HD representation using a Hebbian-like operator and an exponential clamp.

\section{ROBOT RESULTS}

The robot was tested in both a cue poor $(\mathrm{CP})$ and cue rich $(\mathrm{CR})$ environments within a general messy lab environment. Figure 2 shows the $\mathrm{CR}$ environment (a circular arena $\sim 2 \mathrm{~m}$ in diameter). The CP environment was of the same dimensions but with four featureless partitions creating a rectangular box. The arena size corresponds proportionally to the size of typical rat testing arenas. For each experiment, the robot randomly wandered the arena learning new views and binding them to encoder driven HD representations. After 5 minutes training, the robot repeatedly localized its orientation at random spots in the arena selected by the operator. Localization was performed through an accumulative means where each observed view contributes, via its learnt bindings, to the estimated HD distribution with adjustments for movement via encoder feedback. The peak of the distribution was compared to the encoder generated ground truth.

The robot typically learnt $\sim 10$ views in the $\mathrm{CP}$ environment and $\sim 30$ views in the $\mathrm{CR}$ environment, where the views were spread over the full range of orientations. Some views were spurious (less than 10\%) due to the simplistic allocation mechanism however most were significant features (vertical edges) that one would expect to be able to orient off. Accuracy wise, the robot performed well. Figure 2 shows the comparison between the localization heading and the ground truth orientation as the robot rotated on the spot. Over 27 localization steps at 9 randomly selected locations produced a mean error of $12.9^{\circ}\left(\sigma 29^{\circ}\right)$ for the $\mathrm{CR}$ environment and $15^{\circ}\left(\sigma 30^{\circ}\right)$ in the $\mathrm{CP}$ environment. With the $\mathrm{HD}$ resolution at $11.25^{\circ}$ per unit this is a reasonable result.

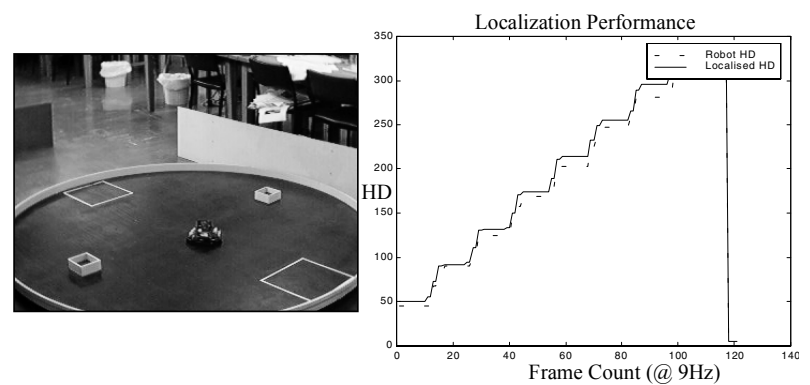

Figure 2. The CR testing environment and localization trail.

\section{DISCUSSION \& CONCLUSIONS}

The results from the robot experiments proved to be robust and repeatable. Within the size of the environments used, which correspond to the typical dimensions of rat arenas, the robot demonstrated that the naïve binding approach is effective for learning orientation. It has yet to be determined if the naïve approach will operate reliably under environmental manipulations however future work will examine this issue.

There are numerous, obvious limitations to the minimalistic approach described here. Firstly, there is no mechanism to correct for the egocentric offset of the observed view. Given the limited field of view of the robot (around $90^{\circ}$ ), the nature of the visual attention mechanism and the coarseness of the HD representation this is not a significant problem. However, future work will attempt to correct this limitation in a plausible fashion.

In conclusion, this paper has described the implementation of a minimalist model of the rat HD system on a mobile robot platform. It was demonstrated that the system was capable of learning allocentric orientation in an unmodified environment in real-time within the limitations of the available computational resources, which proves some validation to the simplistic model of the rat navigation system. Much work remains, however, to develop a fully proficient robot system capable of reproducing ratlike navigation behavior.

\section{REFERENCES}

[1] Browning, B, \& Chang, M. Developing low cost soccer playing robots with on-board vision. International Conference on Robots and Systems (IROS'2001), submitted.

[2] Gaussier, P., et al. Visual navigation in an open environment without a map. The 1997 IEEE/RSJ International Conference on Robot and Systems (IROS'97), (1997), New York, USA.

[3] Redish, A.D. Beyond the Cognitive Map: From place cells to episodic memory, MIT Press, Cambridge, MA, (1999).

[4] Skaggs, W., Knierim, J., Kudrimoti, H., McNaughton, B. A model of the neural basis of the rat's sense of direction. Tesauro, G., et al. (Eds). Advances in Neural Information Processing Systems 7, (1995), 173-180. 